\title{
FDTD Analysis of a Probe-Fed Dielectric Resonator Antenna in Rectangular Waveguide
}

\author{
Yizhe Zhang, Student Member, IEEE, Ahmed A. Kishk, Fellow, IEEE, Alexander B. \\ Yakovlev, Senior Member, IEEE, and Allen W. Glisson, Fellow, IEEE
}

\begin{abstract}
Dielectric resonator antennas (DRA) are characterized for operation in a guided-wave environment with the ultimate goal of their use in modeling of waveguide-based DRA amplifier arrays for spatial power combining. Performance of a single probe-fed DRA element in rectangular waveguide is analyzed by varying design parameters of the DRA and the feeding probe to optimize the structure for the scattering characteristics (port matching and coupling). The effect of hard walls on the DRA behavior is also studied. The numerical analysis of waveguide-based DRA elements is based on the Finite-Difference Time-Domain (FDTD) method and a coaxial probe is modeled by a thin wire approximation implemented in the FDTD algorithm. The numerical results are compared with those generated by using commercial software and exhibit a very good agreement.
\end{abstract}

Index Terms-Dielectric resonator antenna, rectangular waveguide, hard wall, Finite-Difference Time-Domain method.

\section{INTRODUCTION}

D IELECTRIC resonator antennas (DRA) became potentially useful as antenna elements a few decades ago [1]. The DRA's have several attractive features, such as small size, light weight, inherently wideband nature, high radiation ef cienc $y$, and high power handling capability as compared with microstrip antennas, which suffer from higher conduction loss and surface waves in antenna array applications. The use of additional structural parameters (shape, volume, etc.) and a wide range of permittivities makes the design of DRA's more e xible. In addition, different feeding mechanisms can be used for the excitation of DRA elements, which allows for control of DRA input impedance, bandwidth, and radiation pattern. This includes the excitation by slots, probes, microstrip lines, coplanar lines, and dielectric image guides [2]-[4]. Also, the use of dielectric materials with high permittivity in DRA's enables enhancement of the radiation resistance of electrically short probes and loops [5]. Subsequently, systematic theoretical and experimental investigations of DRA's have been reported in [6]-[12] and extensive results of DRA's of cylindrical [1], [3], [8]-[10], rectangular [6], and spherical [7] shapes have been published.

In this paper, coaxial probe-fed DRA elements are studied for operation in a rectangular waveguide with the ultimate goal of their use in waveguide-based DRA ampli er arrays for spatial power combining. Traditionally, the spatial power combiner is formed by an array of amplifying unit cells, with

The authors are with the Department of Electrical Engineering, University of Mississippi, University, MS 38677, USA (e-mail:zyz@olemiss.edu; ahmed@olemiss.edu; yakovlev@olemiss.edu; aglisson@olemiss.edu). each cell receiving, amplifying, and then radiating a signal into free space [13]-[15]. The key challenges of spatial power combining design are the modeling of receive/transmit antenna elements and the uniform excitation of the antenna arrays. Thus, modeling of one element in rectangular waveguide is important to fully understand the behavior of the DRA in the waveguide environment. Regarding the uniform excitation of ampli er arrays, hard electromagnetic walls have been recently realized by dielectric loading along narrow sides of the waveguide to obtain a uniform eld distribution in the waveguide cross-section [16], [17]. The interaction between the DRAs and the hard walls needs to be studied since it signi cantly affects the resonance frequency and matching characteristics of the DRA.

Here, a single probe-fed DRA element is rst investigated for operation in a rectangular waveguide. The scattering parameters are studied by varying geometrical and material parameters of the DRA and the coaxial probe feed. Then, the effect of a dielectric-loaded waveguide on the DRA performance is analyzed. The FDTD method [18], [19] is chosen for the numerical analysis since it provides a full-wave solution of the electrically large and complicated structures, which include different dielectric materials. In the proposed FDTD algorithm, DRA elements and the rectangular waveguide are discretized by using a traditional Yee-cell gridding and the coaxial probe is modeled by a thin wire approximation. The scattering parameters are computed using the FDTD method and compared with the results obtained using the commercial software QuickWave3D [20] and HFSS [21].

The paper is organized as follows. In Section II, a brief description of the FDTD method used in the numerical analysis of waveguide-based probe-fed DRA's is presented. In Section III, an initial design of a single free-space DRA element is presented, followed by the analysis of DRA behavior in an open-ended rectangular waveguide using WIPL-D [22]. Next, the developed FDTD algorithm is used for the parametric analysis of a probe-fed DRA element in a rectangular waveguide to optimize the structure for the scattering characteristics (port matching and coupling). Section IV presents the analysis of a dielectric-loaded waveguide and the effect of hard walls on the DRA performance. Conclusions are summarized in Section V.

\section{THEORY}

The structure to be analyzed here consists of a coaxial probe-fed DRA element inside a rectangular waveguide as shown in Fig. 1. The geometry is analyzed by using the FDTD 
method, wherein the DRA element and rectangular waveguide are discretized by traditional Yee cells. A sinusoidally modulated Gaussian pulse is used for the excitation, and the PML absorbing boundary [23] is implemented to terminate both the waveguide port [24] and the coaxial line port. The scattering parameters are calculated in terms of port voltages normalized by characteristic impedances of the waveguide and coaxial probe, respectively. The S-parameters of the waveguide section with coaxial probe-fed DRA are given by



Fig. 1. Geometry of a waveguide-based coaxial probe-fed DRA.

$$
\begin{array}{r}
S_{11}=\frac{V_{w}^{-}}{V_{w}^{+}}=\frac{V_{w}^{\text {total }}-V_{w}^{+}}{V_{w}^{+}} \\
S_{21}=\frac{V_{c}^{+}}{V_{w}^{+}} \sqrt{\frac{Z_{w}}{Z_{c}}}
\end{array}
$$

where $V_{w}^{-}$is the voltage calculated from the re ection eld at the waveguide port, $V_{w(c)}^{+}$is the voltage calculated from the incident eld at the waveguide (coaxial) port, $V_{w}^{\text {total }}$ is the voltage obtained from the total (incident plus re ected) eld at the waveguide port, and $Z_{w(c)}$ is the characteristic impedance of the waveguide (coaxial line), given by

$$
\begin{array}{r}
Z_{w}=\frac{\omega \mu}{\beta}=\frac{\omega \mu c}{\sqrt{\omega^{2}-\omega_{c}^{2}}} \\
Z_{c}=\frac{V_{k} e^{-j \omega \Delta t / 2}}{\sqrt{I_{k-1} I_{k}}} .
\end{array}
$$

Here, $c$ is the velocity of light, $\beta$ and $\omega_{c}$ are the phase constant and cutoff frequency of the dominant mode of rectangular waveguide, and $V_{k}$ and $I_{k}$ are the voltage and current of the $k$ th cell in the coaxial line. Since both the electric and magnetic elds are separated by half a cell in space and time, the geometric average of two currents $\left(I_{k-1}\right.$ and $\left.I_{k}\right)$ is taken to compensate for the space difference, and a time delay (exponential term in (2)) is introduced to compensate the difference in time [25].

The time-domain modal voltage in the rectangular waveguide can be calculated from the total electric eld as the following integral over the waveguide cross-section $a \times b$

$$
V\left(z_{0}, t\right)=\int_{0}^{b} \int_{0}^{a} \vec{E}\left(x, y, z_{0}, t\right) \cdot \vec{e}(x, y) d x d y
$$

where $V\left(z_{0}, t\right)$ is the time-domain modal voltage of the dominant mode at $z=z_{0}, \vec{E}\left(x, y, z_{0}, t\right)$ is the total time-domain electric eld in the waveguide cross-section, and $\vec{e}(x, y)$ is the electric- eld vector wave function of the dominant mode [26].

The integral over the waveguide cross-section in (3) can be discretized as follows (Fig. 2),

$$
\begin{aligned}
V\left(z_{0}, t\right) & =\sum_{i=1}^{N_{x}} \int_{0}^{b} \int_{x_{i-1}}^{x_{i}} E_{x}\left(x_{i}, y, z_{0}, t\right) e_{x}(x, y) d x d y \\
& +\sum_{j=1}^{N_{y}} \int_{y_{j-1}}^{y_{j}} \int_{0}^{a} E_{y}\left(x, y_{j}, z_{0}, t\right) e_{y}(x, y) d x d y
\end{aligned}
$$

where $N_{x}$ and $N_{y}$ are the numbers of cells in the $x-$ and $y$-direction, respectively. The components of electric- eld vector wave function can be written in the following form by separating the $x-$ and $y$-variables

$$
\begin{aligned}
& e_{x}(x, y)=C_{x} \phi_{x}(x) \phi_{x}(y) \\
& e_{y}(x, y)=C_{y} \phi_{y}(x) \phi_{y}(y)
\end{aligned}
$$

where $C_{x}$ and $C_{y}$ are constants.

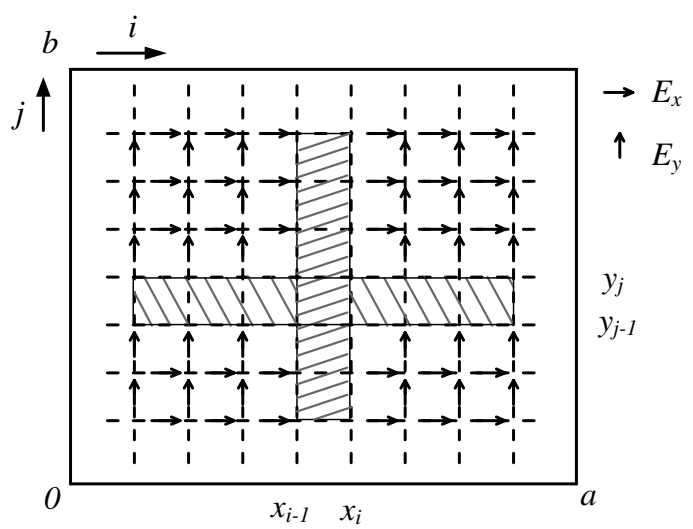

Fig. 2. Discretization of waveguide cross-section in the calculation of voltage.

Substituting equations (5) into equation (4), we obtain

$V\left(z_{0}, t\right)=C_{x} \sum_{i=1}^{N_{x}} \int_{0}^{b} E_{x}\left(x_{i}, y, z_{0}, t\right) \phi_{x}(y) d y \int_{x_{i-1}}^{x_{i}} \phi_{x}(x) d x$
$+C_{y} \sum_{j=1}^{N_{y}} \int_{0}^{a} E_{y}\left(x, y_{j}, z_{0}, t\right) \phi_{y}(x) d x \int_{y_{j-1}}^{y_{j}} \phi_{y}(y) d y$

where the integrals in (6) can be discretized as follows,

$$
\begin{aligned}
& \int_{0}^{b} E_{x}\left(x_{i}, y, z_{0}, t\right) \phi_{x}(y) d y \\
= & \sum_{j=1}^{N_{y}} \int_{y_{j-1}}^{y_{j}} E_{x}\left(x_{i}, y, z_{0}, t\right) \phi_{x}(y) d y
\end{aligned}
$$




$$
\begin{gathered}
=\sum_{j=1}^{N_{y}} \frac{\Delta y_{j}}{2}\left[E_{x}\left(x_{i}, y_{j}, z_{0}, t\right) \phi_{x}\left(y_{j}\right)\right. \\
\left.\quad+E_{x}\left(x_{i}, y_{j-1}, z_{0}, t\right) \phi_{x}\left(y_{j-1}\right)\right], \\
\quad \int_{0}^{a} E_{y}\left(x, y_{j}, z_{0}, t\right) \phi_{y}(x) d x \\
=\sum_{i=1}^{N_{x}} \int_{x_{i-1}}^{x_{i}} E_{y}\left(x, y_{j}, z_{0}, t\right) \phi_{y}(x) d x \\
=\sum_{i=1}^{N_{x}} \frac{\Delta x_{i}}{2}\left[E_{y}\left(x_{i}, y_{j}, z_{0}, t\right) \phi_{y}\left(x_{i}\right)\right. \\
\left.\quad+E_{y}\left(x_{i-1}, y_{j}, z_{0}, t\right) \phi_{y}\left(x_{i-1}\right)\right] .
\end{gathered}
$$

Integrals $\int_{x_{i-1}}^{x_{i}} \phi_{x}(x) d x$ and $\int_{y_{j-1}}^{y_{j}} \phi_{y}(y) d y$ in (6) are calculated in closed form.

The coaxial line is modeled by rectangular prisms instead of two concentric cylinders, since the amount of power travelling into the coaxial line is mainly dependent on the characteristic impedance of the coaxial line [27] and weakly dependent on its speci c shape. The inner conductor of the coaxial line can be represented either by a thin wire or by a number of cells. In our work the inner conductor is approximated as a thin wire with radius smaller than half of the FDTD mesh size [28]. With $E_{z}(i, j, k)=0$ along the wire axis, the spatial dependence of magnetic elds in the vicinity of the wire can be calculated by

$$
\begin{aligned}
H_{y}^{n+\frac{1}{2}}(i, j, k) & =H_{y}^{n-\frac{1}{2}}(i, j, k) \\
& +\frac{\Delta t}{\mu \Delta z}\left[E_{x}^{n}(i, j, k)-E_{x}^{n}(i, j, k+1)\right] \\
& +\frac{2 \Delta t}{\mu \Delta x \ln \left(\frac{\Delta x}{r_{0}}\right)} E_{z}^{n}(i+1, j, k), \\
H_{x}^{n+\frac{1}{2}}(i, j, k) & =H_{x}^{n-\frac{1}{2}}(i, j, k) \\
& +\frac{\Delta t}{\mu \Delta z}\left[E_{y}^{n}(i, j, k+1)-E_{x}^{n}(i, j, k)\right] \\
& -\frac{2 \Delta t}{\mu \Delta y \ln \left(\frac{\Delta y}{r_{0}}\right)} E_{z}^{n}(i, j+1, k),
\end{aligned}
$$

where the wire radius $r_{0}$ is assumed to be less than half of the cell size.

\section{SingLE DRA ELEMENT IN RECTANGULAR WAVEGUIDE}

Our investigation begins with information available in the literature and then we learn step by step about the DRA in different environments. Therefore, we start with a DRA in free space and end up with a DRA in a waveguide loaded with hard walls. Since the FDTD method is one of the methods used in the analysis, the rectangular DRA is a geometry that can be easily analyzed. A single, probe-fed rectangular DRA placed on an in nite ground plane and radiating in free space, as shown in Fig. 3(a), is considered rst, and is analyzed by commercial Method of Moments (MoM) software WIPL-D (a)

$$
\text { Infinite ground plane }
$$
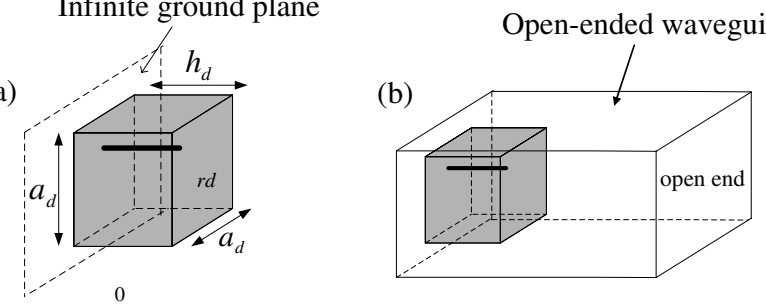

Fig. 3. (a) Geometry of in nite conductor-backed, probe-fed DRA; (b) Geometry of probe-fed DRA inside semi-in nite waveguide.

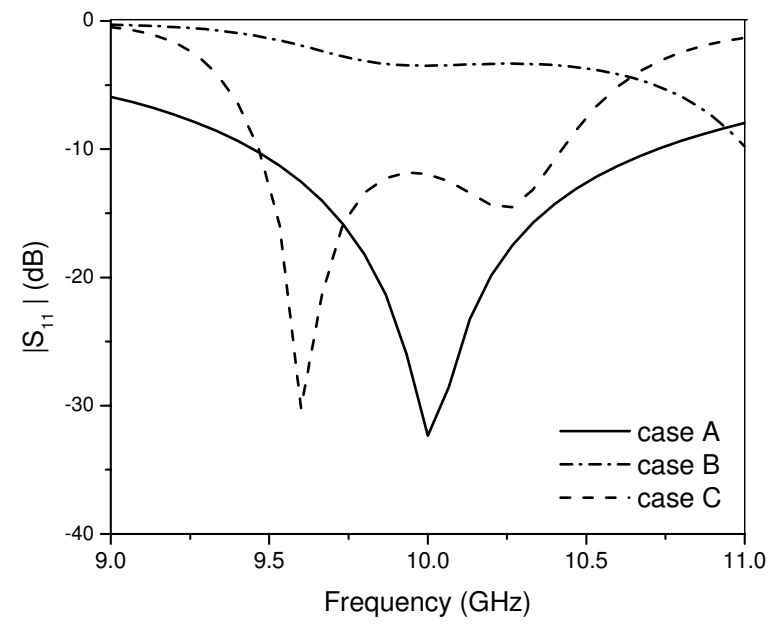

Fig. 4. Re ection coef cient for different con gurations of the DRA. Case A: DRA of Fig. 3(a); Case B: DRA with parameters of Fig. 3(a) placed in waveguide as in Fig. 3(b); Case C: DRA in waveguide with $h_{d}=9.5 \mathrm{~mm}$ and $\delta_{d}=1.5 \mathrm{~mm}$ as in Fig. 3(b).

[22]. The initial dimensions of the DRA structure $a_{d}=5.0$ $\mathrm{mm}, h_{d}=12.0 \mathrm{~mm}, \varepsilon_{r d}=12.0$ are chosen so that the resonant frequency of the $\mathrm{TE}_{11 \delta}$ mode is centered around 10 $\mathrm{GHz}$ [29]. The probe length $l_{d}$ is $4 \mathrm{~mm}$, the probe axis is offset by $\delta_{d}=1.0 \mathrm{~mm}$ from the waveguide centerline in the vertical direction, and the probe radius $r_{w}$ is $0.3 \mathrm{~mm}$. Then, the probe-fed DRA with dimensions from case A (Fig. 3(a)) was placed into an open-ended standard X-band waveguide with cross-sectional dimensions $a=22.86 \mathrm{~mm}$ and $b=10.16$ $\mathrm{mm}$ (case B with geometry shown in Fig. 3(b)).

Figs. 4 shows dispersion behavior of the re ection coef cient for the geometries shown in Figs. 3(a) and 3(b) (cases A and B in Fig. 4). Due to placement of the DRA inside of the rectangular waveguide, the resonant frequency of the DRA in case $B$ is shifted to a frequency higher than $11 \mathrm{GHz}$, which is out of frequency band of interest. Subsequently, the height of the DRA and the probe position were tuned to $h_{d}=9.5 \mathrm{~mm}$ and $\delta_{d}=1.5 \mathrm{~mm}$, respectively, and results are presented as case C. Considerable changes of the resonant frequency and bandwidth are observed in Fig. 4. These dimensions serve as starting values for a numerical study that follows.

An analysis of the waveguide-based coaxial probe-fed DRA (Fig. 1) with the parameters from case $\mathrm{C}$ is performed by the full-wave FDTD method described in Section II. For the rectangular coax of radius $1.2 \mathrm{~mm}$ and lled with the dielectric 


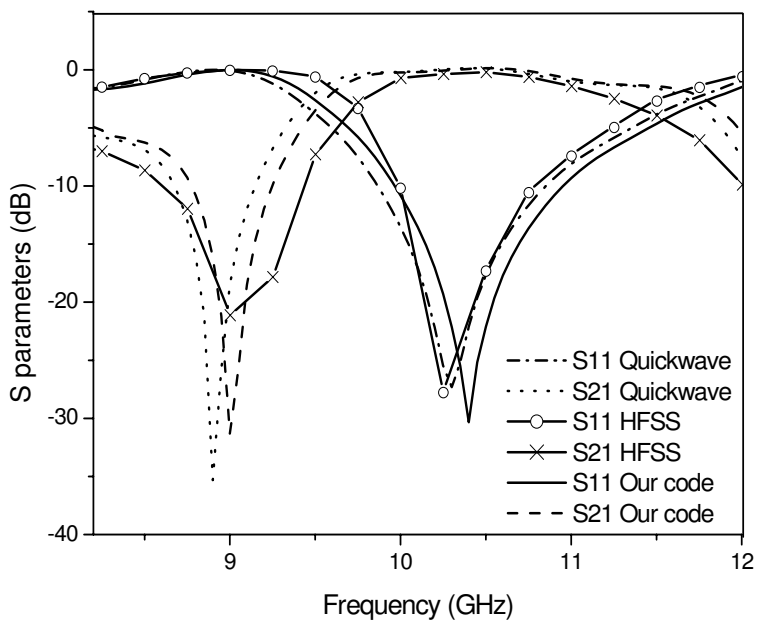

Fig. 5. S-parameters for the waveguide-based probe-fed DRA ( $a_{d}=5.0 \mathrm{~mm}$, $\left.h_{d}=9.5 \mathrm{~mm}, \delta_{d}=1.5 \mathrm{~mm}, l_{d}=4.0 \mathrm{~mm}\right)$.

of permittivity $\varepsilon_{r}=2.56$, the characteristic impedance $Z_{0}$ is $51.98 \Omega$, and only the TEM mode is supported as the cutoff frequency of the $\mathrm{TE}_{11}$ coaxial line mode is approximately 26 $\mathrm{GHz}$ [30]. The probe length $l_{d}$ is $4 \mathrm{~mm}$ and the probe position is offset by $\delta_{d}=1.5 \mathrm{~mm}$ from the waveguide centerline in the vertical direction. Fig. 5 shows the dispersion behavior of the S-parameters. The bandwidth for this case, based on $-10 \mathrm{~dB}$ level, is $10 \%$ (compared to the $3 \%$ bandwidth of a microstrip patch antenna used in a similar con guration [31]). The results in Fig. 5 are veri ed with commercial FDTD software QuickWave3D [20] and HFSS [21] and exhibit very good agreement. Once our FDTD code is veri ed, we depend on it in the following analysis.

The effect on port matching and bandwidth of different parameters of the DRA are then investigated. For the structure operating in the X-band, it is found that position and length of the probe both have a signi cant effect on the matching as well as the overall frequency response. Figs. 6(a) and 6(b) show the return loss and insertion loss of the structure with different values of $l_{d}$. It is found that a short probe couples weakly to the DRA. As the probe length increases, the coupling is increased and a shift to lower resonant frequency is observed. When the probe lengths equal $3.5 \mathrm{~mm}$ and $4.0 \mathrm{~mm}$, wide bandwidth and less re ections are achieved, respectively. As the probe is increased to $4.5 \mathrm{~mm}$ and beyond, the coupling to the DRA starts decreasing.

The effect of the probe position $\delta_{d}$ with respect to the waveguide centerline is illustrated in Figs. 7(a) and 7(b), which show the return loss and insertion loss of the structure for different values of $\delta_{d}$ with $l_{d}=4.0 \mathrm{~mm}$. It is found that the maximum $-10 \mathrm{~dB}$ bandwidth occurs when $\delta_{d}$ reaches $2.0 \mathrm{~mm}$ and also good insersion loss is achieved. It is clear that both the probe length and position can be used to tune and control the response for both port matching as well as wide bandwidth.



(a)

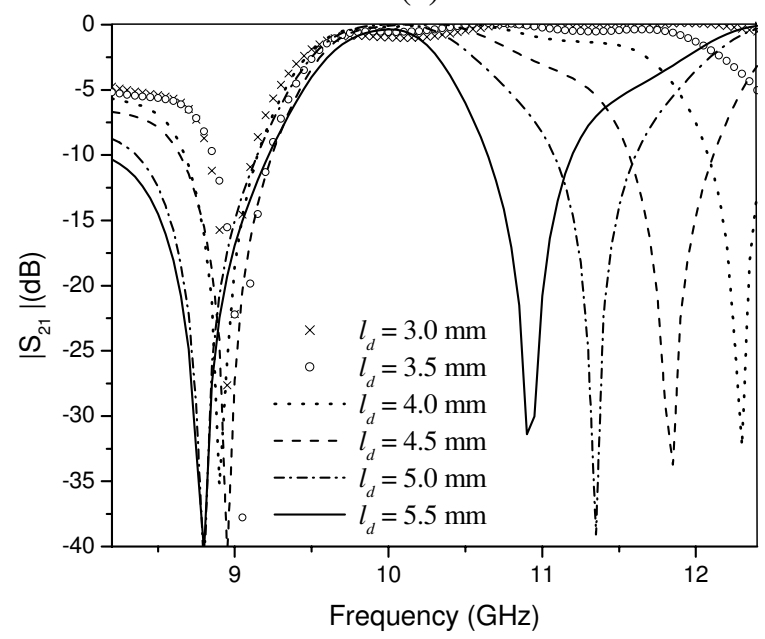

(b)

Fig. 6. Effect of varying the probe length $l_{d}$ in the DRA on (a) return loss and (b) insertion loss, $\left(a_{d}=5.0 \mathrm{~mm}, h_{d}=9.5 \mathrm{~mm}, \delta_{d}=1.5 \mathrm{~mm}\right)$.

\section{Single DRA ELEMENT IN DiEleCtric LOADED RECTANGULAR WAVEGUIDE}

The ultimate goal of this work is to design an ef cient spatial power combining system which needs to achieve a uniform power division among the antenna elements in the DRA array by using hard walls. For one element design, hard electromagnetic walls in the hollow rectangular waveguide can be created by loading its narrow walls with dielectric material. By appropriately choosing the dielectric thickness for a given dielectric material, a uniform eld distribution can be achieved over the cross-section of the waveguide. In a standard X-band waveguide, the PEC boundary de nes the $\mathrm{TE}_{10}$ mode of propagation. The dielectric material loading along the sidewalls in the rectangular waveguide changes the boundary condition so that the $\mathrm{LSE}_{10}$ mode will propagate. The $\mathrm{LSE}_{10}$ mode provides a uniform eld distribution in the inner waveguide region (in between dielectric slabs) when the dielectric thickness $d$ is calculated according to the following 


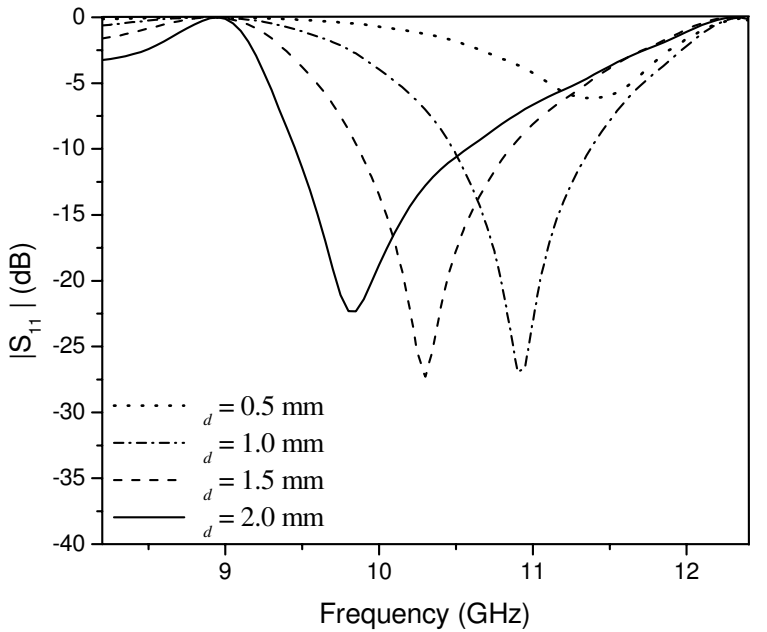

(a)

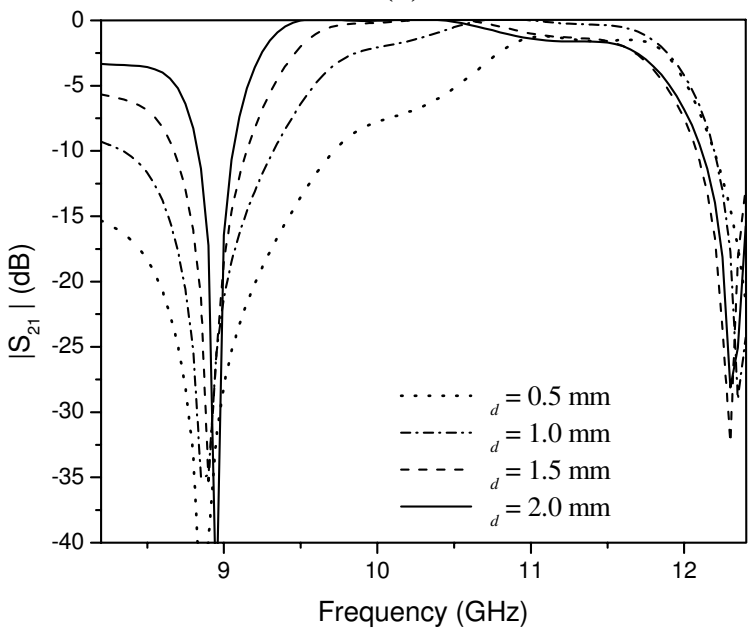

(b)

Fig. 7. Effect of varying the probe position $\delta_{d}$ with respect to the DRA centerline on (a) return loss and (b) insertion loss, $\left(a_{d}=5.0 \mathrm{~mm}, h_{d}=\right.$ $\left.9.5 \mathrm{~mm}, l_{d}=4.0 \mathrm{~mm}\right)$.

formula [32]:

$$
d=\frac{\lambda}{4 \sqrt{\varepsilon_{r}-1}}
$$

where $\lambda$ is the wavelength in free space and $\varepsilon_{r}$ is the relative permittivity of the dielectric material. Hence, at the center frequency of operation, the thickness of the dielectric wall is approximately $\lambda_{d} / 4$, where $\lambda_{d}$ is the wavelength in the dielectric. In Fig. 8, the electric eld amplitudes for the $\mathrm{LSE}_{10}$ mode of the dielectric loaded waveguide and the $\mathrm{TE}_{10}$ mode of the hollow waveguide are plotted along the $x$-axis at 10 $\mathrm{GHz}$. Both of these amplitudes are normalized to unity power.

Since the eld distribution for the $\mathrm{TE}_{10}$ mode of operation in a standard X-band rectangular waveguide is sinusoidal in the waveguide cross-section as shown in Fig. 8, the electric eld in the middle of the waveguide is stronger than that offset from the centerline. Consequently, the coaxial probefed DRA couples more energy when it is placed in the middle



Fig. 8. The electric eld magnitude of the $\mathrm{TE}_{10}$ mode in a hollow rectangular waveguide and the $\mathrm{LSE}_{10}$ mode in rectangular waveguide with hard walls at $10 \mathrm{GHz}$.

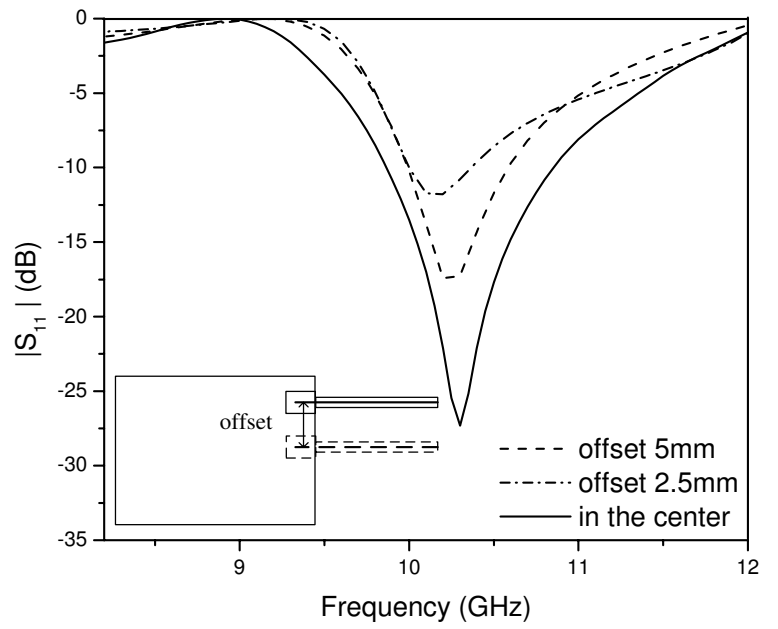

(a)



(b)

Fig. 9. (a) return loss and (b) insertion loss of the DRA element offset from the centerline of the waveguide, $\left(a_{d}=5.0 \mathrm{~mm}, h_{d}=9.5 \mathrm{~mm}, \delta_{d}=1.5 \mathrm{~mm}\right.$, $\left.l_{d}=4.0 \mathrm{~mm}\right)$. 
of the waveguide. As seen in Figs. 9(a) and 9(b), the scattering parameters are obtained for the cases when the DRA is placed in three different positions: in the rst case the DRA is in the middle of waveguide, the second and the third cases are for the DRA placed $2.5 \mathrm{~mm}$ and $5 \mathrm{~mm}$ offset from the waveguide centerline in the horizontal direction, respectively. Figs. 10(a) and $10(\mathrm{~b})$ show the eld distributions (at $10 \mathrm{GHz}$ and $\lambda / 2$ away from the surface of the DRA) for the coaxial probe-fed DRA in the center of the waveguide and $5 \mathrm{~mm}$ offset from the centerline, respectively. In Fig. 10(b), 8 dB power difference between the middle and $5 \mathrm{~mm}$ offset can be observed from the magnitude of the eld. These results verify that the element closest to the side wall of the waveguide will couple less power from the source than the one in the middle.



(a)

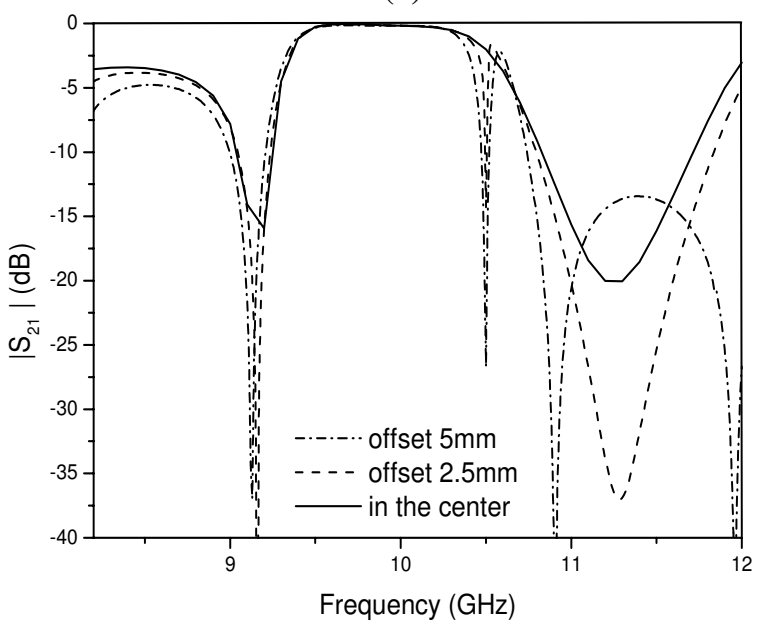

(b)

Fig. 10. (a) return loss and (b) insertion loss of the DRA element offset from the centerline of the waveguide loaded with hard walls, $\left(a_{d}=5.0 \mathrm{~mm}\right.$, $\left.h_{d}=7.5 \mathrm{~mm}, \delta_{d}=2.0 \mathrm{~mm}, l_{d}=3.5 \mathrm{~mm}\right)$.

As seen in the inset in Fig. 11(a), a coaxial probe-fed DRA is placed in the rectangular waveguide with dielectric hard walls. The approximate depth of the hard wall, $d=6.48 \mathrm{~mm}$, is calculated by Eq. (10) and the length of the hard wall $L$ equals to $30 \mathrm{~mm}$. The choice of dielectric material affects the bandwidth of the uniform eld. It has been found that dielectrics with permittivities from 1.2 to 2.2 can be used to achieve a good uniform eld distribution across the waveguide aperture [32] and, therefore, $\varepsilon_{r}=2.2$ is used in our analysis. The coaxial probe-fed DRA element is placed in three different positions: in the middle of waveguide, and $2.5 \mathrm{~mm}$ and 5 $\mathrm{mm}$ offset from the waveguide centerline. Figs. 10(a) and 10(b) demonstrate that the resonance frequency and scattering parameters of these three cases do not change signi cantly as compared to the cases of DRA in the waveguide without hard walls (Figs. 9(a) and 9(b)). The eld distributions (at $10 \mathrm{GHz}$ and $\lambda / 2$ away from the surface of the DRA) for the coaxial probe-fed DRA in the center of the waveguide loaded with hard walls and $5 \mathrm{~mm}$ offset from the centerline are shown in Figs. 12(a) and 12(b). One can see (Fig. 12(b)) that $3 \mathrm{~dB}$ power difference between the middle and $5 \mathrm{~mm}$ offset is achieved, so the DRA element couples approximately the same energy around the center frequency for these three different positions, which veri es that the uniform eld distribution is achieved across the waveguide aperture as shown in Fig. 8.

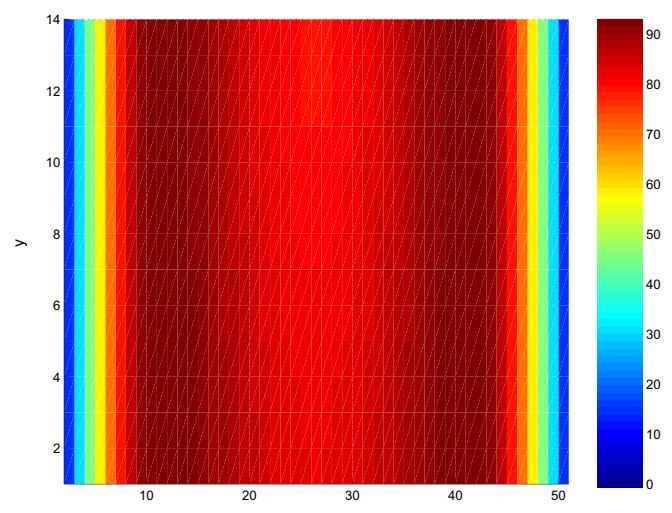

(a)

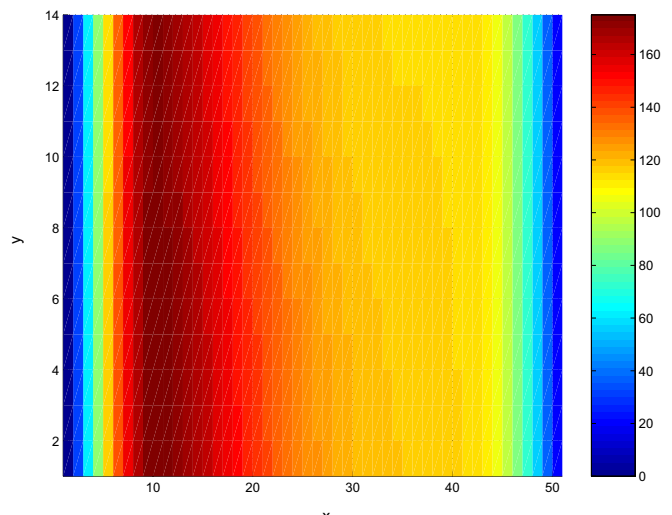

(b)

Fig. 11. Field distribution of the DRA element; (a) in the center of the harded waveguide and (b) offset $5 \mathrm{~mm}$ from the centerline of the waveguide loaded with hard walls, $\left(a_{d}=5.0 \mathrm{~mm}, h_{d}=9.5 \mathrm{~mm}, \delta_{d}=2.0 \mathrm{~mm}, l_{d}=3.5 \mathrm{~mm}\right)$. 


\section{CONCLUSION}

A coaxial probe-fed DRA in a rectangular waveguide excited by the dominant mode was analyzed by using a custom FDTD technique. This analysis provides the necessary information for the optimization of design parameters, such as DRA dimensions and the position and length of the feeding probe. Consequently, $10 \%$ bandwidth was achieved over the frequency band of interest. A waveguide loaded with hard walls was studied to obtain a uniform eld distribution at the waveguide aperture. It is shown that the DRA element couples approximately the same energy around the center frequency when it is placed in the middle of the waveguide and offset from the centerline. This study is a useful step in the extension to the case of the DRA array for increasing the output power and the power combining ef cienc $y$ of waveguide-based spatial power combiner.

\section{ACKNOWLEDGMENT}

This work was partially supported by the National Science Foundation under Grant No. ECS-0220218 and NASA EPSCoR Cooperative Agreement No. NCC5-574.

\section{REFERENCES}

[1] S. A. Long, M. W. McAllister, and L. C. Shen, "The resonant cylindrical dielectric cavity antenna," IEEE Trans. Antennas Propagat., vol. AP-31, pp. 406-412, May 1983.

[2] M. S. Al Salameh and Y. M. M. Antar, "Coplanar-waveguide-fed slotcoupled rectangular dielectric resonator antenna," IEEE Trans. Antennas Propagat., vol. 50, no. 10, pp. 1415-1419, Oct. 2002.

[3] G. P. Junker, A. A. Kishk, and A. W. Glisson, "Input impedance of dielectric resonator antennas excited by a coaxial probe," IEEE Trans. Antennas Propagat., vol. 42, pp. 960-966, July 1994.

[4] G. P. Junker, A. A. Kishk, and A. W. Glisson, "Input impedance of aperture coupled dielectric resonator antennas," IEEE Trans. Antennas Propagat., vol. 44, pp. 600-607, May 1996.

[5] O. Sager and F. Tisi, "On eigenmodes and forced resonance modes of dielectric sphere,” Proc. IEEE, vol. 56, pp. 1593-1594, Sept. 1968.

[6] M. W. McAllister, S. A. Long, and G. L. Conway, "Rectangular dielectric resonator antenna," Electron. Lett., vol. 19, pp. 219-220, Mar. 1983.

[7] M. W. McAllister and S. A. Long, "Resonant hemispherical dielectric antenna," Electron. Lett., vol. 20, pp. 657-659, Aug. 1984.

[8] A. A. Kishk, H. A. Auda, and B. Ahn, "Radiation characteristics of cylindrical dielectric resonator antenna with new applications," IEEE Trans. Antennas Propagat. Soc. Newsletter, vol. 31, pp. 7-16, Feb. 1989.

[9] A. A. Kishk, B. Ahn, and D. Kajfez, "Broadband stacked dielectric resonator antennas," Electron. Lett., vol. 25, pp. 1232-1233, Aug. 1989.

[10] A. A. Kishk, M. R. Zunoubi, and D. Kajfez, "A numerical study of a dielectric disk antenna above grounded dielectric substrate," IEEE Trans. Antennas Propagat., vol. 41, pp. 813-821, June 1993.

[11] A. A. Kishk, Y. Yin, and A. W. Glisson, "Conical dielectric resonator antennas for wideband applications," IEEE Trans. Antennas Propagat., vol. 50, pp. 469-474, Apr. 2002.

[12] A. A. Kishk, "Wideband dielectric resonator antenna in a truncated tetrahedron form excited by a coaxial probe," IEEE Trans. Antennas Propagat., vol. 51, pp. 2913-2917, Oct. 2003.

[13] A. B. Yakovlev, S. Ortiz, M. Ozkar, A. Mortazawi, and M. B. Steer, "A waveguide-based aperture-coupled patch ampli er array - full-wave system analysis and experimental validation," IEEE Trans. Microwave. Theory Tech., vol. 48, no. 12, pp. 2692-2699, Dec. 2000.

[14] S. Ortiz, J. Hubert, L. Mirth, E. Schlecht, and A. Mortazawi, "A highpower Ka-band quasioptical ampli er array," IEEE Trans. Microwave. Theory Tech., vol. 50, no. 2, pp. 487-494, Feb. 2002.

[15] M. Ozkar and A. Mortazawi, "Electromagnetic modeling and optimization of spatial power combiners/dividers with hard horns," IEEE Trans. Antennas Propagat., vol. 53, pp. 144-150, Jan. 2005.

[16] M. A. Ali, S. C. Ortiz, T. Ivanov, and A. Mortazawi, "Analysis and measurement of hard-horn feeds for the excitation of quasioptical ampli ers," IEEE Trans. Microwave Theory Tech., vol. 47, pp. 479-487, Apr. 1999.
[17] M. N. M. Kehn and P. S. Kildal, "Miniaturized rectangular hard waveguides for use in multifrequency phased arrays," IEEE Trans. Antennas Propagat., vol. 53, no. 1, pp. 100-109, Jan. 2005.

[18] K. S. Yee, "Numerical solution of initial boundary value problems involving Maxwell's equations in isotropic media," IEEE Trans. Antennas Propagat., vol. 14, pp. 302-307, 1966.

[19] A. Ta o ve and S. C. Hagness, , Computational Electrodynamics: The Finite-Difference Time-Domain Method, 3rd edition, Norwood, MA: Artech House, 2005.

[20] QuickWave3D: A General Purpose Electromagnetic Simulator Based on Conformal Finite-Difference Time-Domain Method, v. 2.2, QWED Sp. Zo.o, Dec. 1998.

[21] HFSS: High Frequency Structure Simulator Based on the Finite Element Method, v. 9.2.1, Ansoft corporation, 2004.

[22] B. M. Kolundzija, J. S. Ognjanovic, and T. K. Sarkar, WIPL-D: Electromagnetic Modeling of Composite Metallic and Dielectric Structures, Software and User's Manual. Reading, MA: Artech House, 2000.

[23] J. P. Berenger, "A perfectly matched layer for the absorption of electromagnetic waves," J. Comp. Phys., vol. 114, pp. 185-200, 1994.

[24] C. E. Reuter, R. M. Joseph, E. T. Thiele, D. S. Katz, and A. Ta o ve, "Ultrawideband absorbing boundary condition for termination of waveguiding structures in FDTD simulations," IEEE Microwave Guided Wave Lett., vol. 4, pp. 344-346, Oct. 1994.

[25] J. Fang and D. Xeu, "Numerical errors in the computation of impedances by FDTD method and ways to eliminate them," IEEE Microwave Guided Wave Lett., vol. 5, pp. 6-8, Jan. 1995.

[26] R. E. Collin, Field Theory of Guided Waves, 2nd ed. New York: McGraw-Hill, IEEE Press, 1991.

[27] C. Wu, K. -L. Wu, Z. Bi, and J. Litva, "Modeling of coaxial-fed microstrip patch antenna by nite difference time domain method," IEEE Eletrocnics Lett., vol. 27, pp. 1691-1692, Sept. 1991.

[28] K. R. Umashankar, A. Ta o ve, and B. Beker, "Calculation and experimental validation of induced currents on coupled wires in an arbitrary shaped cavity," IEEE Trans. Antennas Propagat., vol. 35, pp. 1248-1257, Nov. 1987.

[29] K. M. Luk and K. W. Leung, Dielectric Resonator Antennas, Hertfordshire, England: Research Studies Press Ltd., 2002.

[30] D. M. Pozar, Microwave Engineering, 2nd ed, NY: Wiley, 1998.

[31] M. Ozkar, Electromagnetic Modeling for the Optimized Design of Spatial Power Ampli ers with Hard Horn Feeds, Ph.D. Dissertation, North Carolina State University, 2001.

[32] G. N. Tsandoulas and W. D. Fitzgerald, "Aperture ef cienc y enhancement in dielectrically loaded horns," IEEE Trans. Antennas Propagat., vol. 20, pp. 69-74, 1972. 


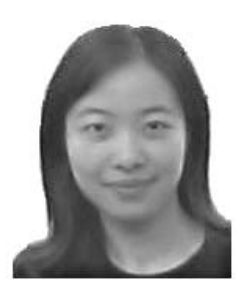

Yizhe Zhang received the B.Sc and M.Sc degrees in electrical engineering from Southeast University, China, in 2000 and 2003, respectively. She is currently working toward the Ph.D. degree in electrical engineering at the University of Mississippi, University.

From 2000 to 2003, she was a Research Assistant in the Department of Electrical Engineering, Southeast University. Her research interests include dielectric resonator antennas, microstrip antennas, numerical methods in electromagnetics, and modeling of high-frequency ampli er arrays for spatial power combining.



Ahmed A. Kishk is a Professor of Electrical Engineering, University of Mississippi (since 1995). He was an Associate Editor of Antennas \& Propagation Magazine from 1990 to 1993 . He is now an Editor of Antennas \& Propagation Magazine. He was a Coeditor of the special issue on Advances in the Application of the Method of Moments to Electromagnetic Scattering Problems in the ACES Journal. He was also an editor of the ACES Journal during 1997. He was an Editor-in-Chief of the ACES Journal from 1998 to 2001. He was the chair of Physics and Engineering division of the Mississippi Academy of Science (2001-2002). $\mathrm{He}$ was a guest Editor of the special issue on arti cial magnetic conductors, soft/hard surfaces, and other complex surfaces, on the IEEE Transactions on Antennas and Propagation, January 2005.

His research interest includes the areas of design of millimeter frequency antennas, feeds for parabolic re ectors, dielectric resonator antennas, microstrip antennas, soft and hard surfaces, phased array antennas, and computer aided design for antennas. He has published over 150 refereed Journal articles and book chapters. He is a coauthor of the Microwave Horns and Feeds book (London, UK, IEE, 1994; New York: IEEE, 1994) and a coauthor of chapter 2 on Handbook of Microstrip Antennas (Peter Peregrinus Limited, United Kingdom, Ed. J. R. James and P. S. Hall, Ch. 2, 1989). Dr. Kishk received the 1995 outstanding paper award for a paper published in the Applied Computational Electromagnetic Society Journal. He received the 1997 Outstanding Engineering Educator Award from Memphis section of the IEEE. He received the Outstanding Engineering Faculty Member of the 1998. He received the Award of Distinguished Technical Communication for the entry of IEEE Antennas and Propagation Magazine, 2001. He received the 2001 Faculty research award for outstanding performance in research. He received the 2005 School of Engineering Senior Faculty Research Award. He received the 2005 Faculty research award for outstanding performance in research. $\mathrm{He}$ also received The Valued Contribution Award for outstanding Invited Presentation, "EM Modeling of Surfaces with STOP or GO Characteristics Arti cial Magnetic Conductors and Soft and Hard Surfaces" from the Applied Computational Electromagnetic Society. He received the Microwave Theory and Techniques Society Microwave Prize 2004. Dr. Kishk is a Fellow member of IEEE since 1998 (Antennas and Propagation Society and Microwave Theory and Techniques), a member of Sigma Xi society, a member of the U.S. National Committee of International Union of Radio Science (URSI) Commission B, a member of the Applied Computational Electromagnetics Society, a member of the Electromagnetic Academy, and a member of $\mathrm{Ph}$ Kappa Phi Society.

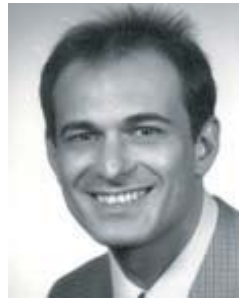

Alexander B. Yakovlev was born in the Ukraine in 1964. He received the Ph.D. degree in Radiophysics from the Institute of Radiophysics and Electronics, National Academy of Sciences, Ukraine, in 1992, and the Ph.D. degree in Electrical Engineering from the University of Wisconsin at Milwaukee, in 1997.

From 1992 to 1994, he was an Assistant Professor with the Department of Radiophysics at Dniepropetrovsk State University, Ukraine. From 1994 to 1997, while working toward his doctorate degree, he was employed as a Research and Teaching Assistant in the Department of Electrical Engineering and Computer Science at the University of Wisconsin in Milwaukee. From 1997 to 1998, he was an R\&D Engineer in Ansoft Corporation/Compact Software Division, Paterson, NJ, and in Ansoft Corporation, Pittsburgh, PA. From 1998 to 2000, he was a Postdoctoral Research Associate with the Electrical and Computer Engineering Department at North Carolina State University, Raleigh, NC. In summer of 2000, he joined the Department of Electrical Engineering at the University of Mississippi as an Assistant Professor, and was promoted to the rank of Associate Professor in 2004.

His research interests include mathematical methods in applied electromagnetics, analysis of arti cial magnetic conductor surfaces and guidedwave structures, modeling of high-frequency interconnection structures and ampli er arrays for spatial and quasi-optical power combining, microstrip and waveguide discontinuities, integrated-circuit elements and devices, theory of leaky waves, transient elds in layered media, catastrophe and bifurcation theories.

Dr. Yakovlev received the Young Scientist Award at the 1992 URSI International Symposium on Electromagnetic Theory, Sydney, Australia, and the Young Scientist Award at the 1996 International Symposium on Antennas and Propagation, Chiba, Japan. In 2003, he received a Junior Faculty Research Award in the School of Engineering at The University of Mississippi. Dr. Yakovlev is an Associate Editor-in-Chief of the ACES Journal and in September 2005 he became an Associate Editor of the IEEE Transactions on Microwave Theory and Techniques. He is a Senior Member of the IEEE (Microwave Theory and Techniques Society and Antennas and Propagation Society) and Member of URSI Commission B. He is coauthor of the book Operator Theory for Electromagnetics: An Introduction, Springer, New York, NY, 2001.

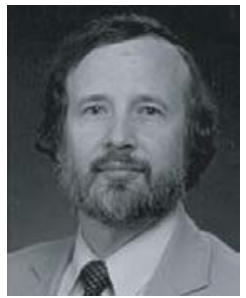

Allen W. Glisson received the B.S., M.S., and Ph.D. degrees in electrical engineering from the University of Mississippi, in 1973, 1975, and 1978, respectively. In 1978, he joined the faculty of the University of Mississippi, where he is currently a Professor and Chair of the Department of Electrical Engineering. He was selected as the Outstanding Engineering Faculty Member in 1986, 1996, and 2004. He received a Ralph R. Teetor Educational Award in 1989 and in 2002 he received the Faculty Service Award in the School of Engineering. His current research interests include the development and application of numerical techniques for treating electromagnetic radiation and scattering problems, and modeling of dielectric resonators and dielectric resonator antennas.

Dr. Glisson is a Fellow of the IEEE, a member of Commission B of the International Union of Radio Science, and a member of the Applied Computational Electromagnetics Society. He has received a best paper award from the SUMMA Foundation, twice received a citation for excellence in refereeing from the American Geophysical Union, and he was a recipient of the 2004 Microwave Prize awarded by the Microwave Theory and Techniques Society. Since 1984, he has served as the Associate Editor for Book Reviews and Abstracts for the IEEE Antennas and Propagation Society Magazine. He has served on the Board of Directors of the Applied Computational Electromagnetics Society, is currently Treasurer of ACES, and is a member of the AP-S IEEE Press Liaison Committee. He has previously served as a member of the IEEE Antennas and Propagation Society Administrative Committee, as the secretary of Commission B of the U.S. National Committee of URSI, as an Associate Editor for Radio Science, as Co-Editor-in-Chief of the Applied Computational Electromagnetics Society Journal, and as the Editor-in-Chief of the IEEE Transactions on Antennas and Propagation. 